Correspondence

Ke-Qin Zhang

kqzhang111@yahoo.com.cn

Zhaohui Meng

zhhmeng@yahoo.cn

Received 12 July 2010

Revised 27 August 2010

Accepted 7 September 2010

\section{Crystal structure and mutagenesis analysis of chitinase CrChi1 from the nematophagous fungus Clonostachys rosea in complex with the inhibitor caffeine}

Jinkui Yang, ${ }^{1} \dagger$ Zhongwei Gan, ${ }^{1,2} \dagger$ Zhiyong Lou, ${ }^{3} \dagger$ Nan Tao, ${ }^{1,4}$ Qili Mi, ${ }^{1}$ Lianming Liang, ${ }^{1}$ Yuna Sun, ${ }^{3}$ Yu Guo, ${ }^{3}$ Xiaowei Huang, ${ }^{1}$ Chenggan Zou, ${ }^{1}$ Zihe Rao, $^{3}$ Zhaohui Meng $^{1,5}$ and Ke-Oin Zhang ${ }^{1}$

${ }^{1}$ Laboratory for Conservation and Utilization of Bio-Resources, and Key Laboratory for Microbial Resources of the Ministry of Education, Yunnan University, Kunming 650091, PR China

${ }^{2}$ Middle School Attached to Yunnan Normal University, Kunming 650106, PR China

${ }^{3}$ Tsinghua-Nankai-IBP Joint Research Group for Structural Biology, Tsinghua University, Beijing 100084, PR China

${ }^{4}$ Institute of Biotechnology, Yunnan Academy of Agricultural Sciences, Kunming, Yunnan 650223, PR China

${ }^{5}$ Laboratory of Molecular Cardiology, Department of Cardiology, The First Affiliated Hospital of Kunming Medical College, Kunming 650032, PR China

\begin{abstract}
Chitinases are a group of enzymes capable of hydrolysing the $\beta$ - $(1,4)$-glycosidic bonds of chitin, an essential component of the fungal cell wall, the shells of nematode eggs, and arthropod exoskeletons. Chitinases from pathogenic fungi have been shown to be putative virulence factors, and can play important roles in infecting hosts. However, very limited information is available on the structure of chitinases from nematophagous fungi. Here, we present the $1.8 \AA$ resolution of the first structure of a Family 18 chitinase from this group of fungi, that of Clonostachys rosea CrChi1, and the $1.6 \AA$ resolution of CrChi1 in complex with a potent inhibitor, caffeine. Like other Family 18 chitinases, CrChi1 has the DXDXE motif at the end of strand $\beta 5$, with Glu174 as the catalytic residue in the middle of the open end of the $(\beta / \alpha)_{8}$ barrel. Two caffeine molecules were shown to bind to $\mathrm{CrChi1}$ in subsites -1 to +1 in the substrate-binding domain. Moreover, sitedirected mutagenesis of the amino acid residues forming hydrogen bonds with caffeine molecules suggests that these residues are important for substrate binding and the hydrolytic process. Our results provide a foundation for elucidating the catalytic mechanism of chitinases from nematophagous fungi and for improving the pathogenicity of nematophagous fungi against agricultural pest hosts.
\end{abstract}

\section{INTRODUCTION}

Chitin, a polymer of $\beta$ - $(1,4)$-linked $N$-acetylglucosamine (GlcNAc), is an essential structural component of fungal cell walls, the shells of nematode eggs, and the exoskeletons of arthropods. Family 18 chitinases (CAZY GH 18), which degrade this polymer, play key roles in the life cycles of pathogenic fungi (Lorito et al., 1996). Fungi can produce chitinases throughout their growth cycle, and these enzymes are believed to contribute to morphogenetic and pathogenic processes, including spore germination, hyphal

†These authors contributed equally to this work.

Abbreviations: CAZY, carbohydrate-active enzymes database; PDB, Protein Data Bank; RMS, root mean square. branching and mycoparasitic interaction (Gooday et al., 1992; Kuranda \& Robbins, 1991; Seidl et al., 2005). For many pathogenic fungi, their chitinases are important virulence factors and promising antifungal targets.

Structural studies of chitinase-inhibitor complexes have provided crucial information on the modes of binding, the specificity of chitinase inhibitors, and the mechanism of the hydrolysis reaction (Terwisscha van Scheltinga et al., 1995; van Aalten et al., 2001). Several chitinase inhibitors have been identified, including allosamidin (Bortone et al., 2002), the cyclic pentapeptides argifin and argadin (Arai et al., 2000; Omura et al., 2000), and 8-chlorotheophylline, kinetin and acetazolamide (Hurtado-Guerrero \& van Aalten, 2007). However, due to their high molecular 
masses and difficulty in synthesis, these inhibitors have limited practical applications. Recently, three xanthine derivatives, theophylline, caffeine and pentoxifylline, were identified as competitive inhibitors of bacterial, fungal, and human Family 18 chitinases (Rao et al., 2005a, b). These xanthine derivatives have low molecular masses and are commercially available. These properties suggest that they might be ideal for developing specific inhibitors of Family 18 chitinases. In addition, site-directed mutagenesis provides a tool for studying the function of amino acid residues and domains. For example, substitution of loop 2 residue N372 with Ala or Gly in $\delta$-endotoxin increased the toxicity of Bacillus thuringiensis to gypsy moth (Lymantria dispar) larvae by eightfold and enhanced its binding affinity to gypsy moth midgut brush border membrane vesicles by fourfold (Rajamohan et al., 1996). Therefore, structural analysis of chitinases may be valuable for analysing the key amino acid residues associated with catalytic domains and for improving the biocontrol potential of the producing organisms by protein engineering. However, to our knowledge, no crystal data for chitinases from nematophagous fungi are yet available.

The filamentous fungus Clonostachys rosea (syn. Gliocladium roseum) is a potential biocontrol agent with a worldwide distribution. Many isolates of $C$. rosea have been studied as biocontrol agents against diverse fungal plant pathogens (Sutton et al., 1997; Xue, 2003). Recently, a chitinase gene, Crchil, was cloned from C. rosea, and its expression level was found to be strongly upregulated by solubilized components (e.g. chitinous components) of the cell wall from the plantpathogenic fungus Rhizoctonia solani (Gan et al., 2007a). Moreover, three endochitinase-encoding genes, $\mathrm{cr}$-ech58, $\mathrm{cr}$ ech42 (orthologous to Crchil analysed here) and cr-ech37, were characterized from $C$. rosea strain IK726 (Mamarabadi et al., 2008). The endochitinase activity was specifically induced in media containing chitin or Fusarium culmorum cell walls as sole carbon source. The expression of $c r$-ech 42 and $c r-e c h 37$ was induced by F. culmorum cell walls and chitin whereas that of cr-ech58 was not affected (Mamarabadi et al., 2008). CrChil showed a high degree of similarity to LpChil in primary amino acid sequence. LpChil was identified from the nematophagous fungus Lecanicillium psalliotae (syn. Verticillium psalliotae) and it could degrade the eggs of the root-knot nematode Meloidogyne incognita (Gan et al., 2007b). These results suggest that chitinase CrChil play a key role during the infection of different hosts by C. rosea (Gan et al., 2007a; Li et al., 2006). In this study, we describe, we believe for the first time, the crystal structure of a chitinase, CrChil, from the nematophagous fungus C. rosea. Furthermore, site-directed mutagenesis was carried out to define the crystal structure of CrChil in complex with the inhibitor caffeine.

\section{METHODS}

Crystallization and data collection. Cloning, expression and purification of chitinase CrChil from C. rosea was described in our recent report (Gan et al., 2009). Purified CrChil was concentrated to $20 \mathrm{mg} \mathrm{ml}{ }^{-1}$ in $20 \mathrm{mM}$ Tris/HCl $(\mathrm{pH} \mathrm{8.0)}$ using an Amicon centrifugal filter device (Millipore). Crystallization was performed by the hanging-drop vapour-diffusion method at $16{ }^{\circ} \mathrm{C}$. Drops consisting of $0.5 \mu \mathrm{l}$ protein solution and $0.5 \mu \mathrm{l}$ reservoir solution were equilibrated against $0.2 \mathrm{ml}$ reservoir solution consisting of $0.2 \mathrm{M}$ ammonium dihydrogen phosphate and $15 \%(\mathrm{w} / \mathrm{v})$ PEG3350. Crystals of CrChil complexed with the inhibitor caffeine were obtained by adding $1 \mu \mathrm{l}$ caffeine $(1.0 \mathrm{mM})$ to the drops which contained the crystals and incubating overnight.

After its formation, the crystal was mounted on a nylon loop and flash-cooled in a stream of nitrogen gas at $100 \mathrm{~K}$ using an Oxford Cryosystems cryostream. Diffraction data were collected on a MAR345dtb (MAR Research) image-plate detector at $100 \mathrm{~K}$ using a Rigaku MM-007 rotating-anode home X-ray generator operating at $40 \mathrm{kV}$ and $20 \mathrm{~mA}(\lambda=1.5418 \AA, 0.154128 \mathrm{~nm})$. All intensity data were indexed, integrated and scaled with the HKL2000 package (Otwinowski \& Minor, 1997).

Structure determination and refinement. The structure of apoCrChil belonged to the $P 2_{1}$ space group and was solved by molecular replacement to $1.8 \AA$ employing the crystal structure of a chitinase [Protein Data Bank (PDB) code: 1W9P] from Aspergillus fumigatus (Rao et al., 2005a) as an initial search model, using the program PHASER (McCoy et al., 2007). The clear solutions in both the rotation and translation functions indicated the presence of one molecule in the asymmetrical unit, which was also suggested by the Matthews coefficient and solvent content (Matthews, 1968). Residues that differed between CrChil and the search model were manually rebuilt using the program Coot (Emsley \& Cowtan, 2004) under the guidance of $F_{\mathrm{o}}-F_{\mathrm{c}}$ and $2 F_{\mathrm{o}}-F_{\mathrm{c}}$ electron-density maps. After the refinement of the model using simulated annealing, energy minimization, restrained individual $B$ factors and the addition of 502 solvent molecules in CNS (Brünger et al., 1998), the respective working $R$ factor and $R_{\text {free }}$ dropped from 0.42 and 0.45 to 0.20 and 0.25 for all data from 50.0 to $1.8 \AA$. Model refinements were monitored by calculating $R_{\text {free }}$ based on a subset containing $10 \%$ of the total reflections. The crystal structure of CrChil complexed with caffeine was solved by molecular replacement using the apo-CrChil structure as the initial search model in PHASER (McCoy et al., 2007), to 1.6 $\AA$. The same refinement procedure was performed as for the apoCrChil structure with manually built bound caffeine into a complex structure using unambiguous $F_{\mathrm{o}}-F_{\mathrm{c}}$ and $2 F_{\mathrm{o}}-F_{\mathrm{c}}$ electron-density maps. Model geometry was verified using the program PROCHECK (Laskowski et al., 1993). Data collection and refinement statistics are detailed in Table 1. All structure figures in this paper were generated by PYMOL (DeLano, 2002), MOLSCRIPT (Esnouf, 1997) and Raster3D (Merritt \& Murphy, 1994).

Enzymology and mutagenesis. Chitinase activity was measured using the fluorogenic substrate 4-methylumbelliferyl $\beta$-D- $N, N^{\prime}, N^{\prime \prime}$ triacetylchitotrioside; Sigma) in a standard assay, as by Rao et al. (2005a). Briefly, in a final volume of $100 \mu \mathrm{l}, 2 \mathrm{nM}$ enzyme was incubated with 5-25 $\mu \mathrm{M}$ substrate in McIlvain buffer ( $100 \mathrm{mM}$ citric acid, $200 \mathrm{mM}$ sodium phosphate $\mathrm{pH} 5.5$ ) containing $0.1 \mathrm{mg}$ BSA $\mathrm{ml}^{-1}$, for $10 \mathrm{~min}$ at $37^{\circ} \mathrm{C}$. After the addition of $50 \mu \mathrm{l} 3 \mathrm{M}$ glycine/ $\mathrm{NaOH}$ ( $\mathrm{pH}$ 10.3), the fluorescence of the liberated 4-methylumbelliferone was quantified using a SPECTRAmax GEMINI XS instrument (Molecular Devices), with excitation and emission wavelengths of $360 \mathrm{~nm}$ and $455 \mathrm{~nm}$, respectively. Experiments were performed in triplicate. Production of 4-methylumbelliferone was linear with time for the tested incubation period, and less than $10 \%$ of the available substrate was hydrolysed.

To determine the action mode of caffeine on CrChil, the inhibitor affinity was first estimated by determining $\mathrm{IC}_{50}$ values in a standard assay, as described above. The $K_{\mathrm{i}}$ values were approximated by 
Table 1. Data collection and refinement statistics

\begin{tabular}{|c|c|c|}
\hline Crystallographic data & Apoenzyme & Complexed with caffeine \\
\hline Space group & $P 2_{1}$ & $P 2_{1}$ \\
\hline Cell parameters & $\begin{array}{l}a=44.1 \AA, b=71.7 \AA, c=59.1 \AA \text {, } \\
\alpha=\gamma=90^{\circ}, \beta=91.3^{\circ}\end{array}$ & $\begin{array}{l}a=44.2 \AA, b=72.0 \AA \\
c=59.3 \AA, \alpha=\gamma=90^{\circ}, \beta=91.2^{\circ}\end{array}$ \\
\hline Resolution range $(\AA)$ & $50.0-1.8(1.9-1.8)^{\star}$ & $50.0-1.6(1.7-1.6)^{\star}$ \\
\hline Total reflections & 126163 & 133236 \\
\hline Unique reflections & 34080 & 40969 \\
\hline Completeness (\%) & $97.7(92.3)^{*}$ & $88.2(96.1)^{*}$ \\
\hline$R_{\text {merge }}(\%) \dagger$ & $8.1(48.0)^{*}$ & $4.3(20.4)^{*}$ \\
\hline$I / \sigma$ & $17.7(2.3)^{*}$ & $18.9(5.1)^{*}$ \\
\hline \multicolumn{3}{|l|}{ Refinement statistics } \\
\hline No. of reflections used & 30889 & 38880 \\
\hline No. of reflections in test set & 1561 & 3046 \\
\hline$R$ factor $(\%) \ddagger$ & 20.3 & 18.0 \\
\hline$R_{\text {free }}(\%) \ddagger$ & 26.0 & 23.1 \\
\hline \multicolumn{3}{|l|}{ RMS deviation } \\
\hline Bonds $(\AA)$ & 0.017 & 0.012 \\
\hline Angles $\left({ }^{\circ}\right)$ & 1.225 & 1.349 \\
\hline Mean B factor & 21.2 & 13.8 \\
\hline \multicolumn{3}{|l|}{ Ramachandran plot } \\
\hline Residues in most favoured regions & 95.4 & 90.2 \\
\hline Residues in additional allowed regions & 4.6 & 9.5 \\
\hline
\end{tabular}

${ }^{\star}$ Numbers in parentheses correspond to the highest-resolution shell.

$\dagger R_{\text {merge }}=\Sigma h \Sigma I I I_{i h}-<I_{h}>\mid / \Sigma h \Sigma I<I_{\mathrm{h}}>$, where $<I_{h}>$ is the mean intensity of the observations $I_{i h}$ of reflection $h$. $\ddagger R$ factor $=\Sigma\left(|| F_{\text {obs }}|-| F_{\text {calc }}||\right) / \Sigma\left|F_{\text {obs }}\right| ; R_{\text {free }}$ is the $R$ factor for a subset $(5 \%)$ of reflections that was selected for prior refinement calculations and not included in the structure refinement.

determining the kinetic parameter $K_{\mathrm{m}}$ in the presence of the inhibitor at a concentration close to the $\mathrm{IC}_{50}$. The mode of action was determined by plotting the data as Lineweaver-Burk plots, and by fitting all data to the standard competitive inhibition equation.

CrChil mutants were constructed using the MutantBEST kit from Takara (Japan). The DNA sequences of mutated gene fragments were confirmed using an ABI PRISM 377 DNA sequencer (Applied Biosystems), and mutant genes were cloned and expressed in Escherichia coli BL21(DE3) following the protocols described above. Mutant proteins were purified in the same manner as the wild-type. The renaturation of inclusion bodies followed the procedure described in a previous report (Meng et al., 2006).

Accession numbers. Crystallographic coordinates and structure factors have been deposited in the RCSB Protein Data Bank with accession codes 3G6L (apo-CrChi1) and 3G6M (complex).

\section{RESULTS AND DISCUSSION}

\section{Overall structure of CrChi1}

The structures of the $44 \mathrm{kDa}$ form of apo-CrChil and in complex with its inhibitor caffeine were solved by molecular replacement and refined against $1.8 \AA$ and $1.6 \AA$ diffraction data to $R$ factors of $20 \%$ and $18 \%$ $\left(R_{\text {free }}=26 \%\right.$ and $\left.23 \%\right)$, respectively. The structure of apoCrChil consists of two parts: eight strands of parallel $\beta$ barrels are surrounded by eight $\alpha$-helices to form the core domain (Fig. 1). The core domain, which was named as a $(\beta / \alpha)_{8}$ TIM barrel, has been observed in other Family 18 chitinases from A. fumigatus (afChi) (PDB code: 1W9P) (Rao et al., 2005a), Coccidioides immitis (cmChi) (PDB code: 1D2K) (Hollis et al., 2000), human chitotriosidase (huChi) (PDB code: 1LG1) (Fusetti et al., 2002), and chitinases A (chiA) (PDB code: 1EDQ) (Papanikolau et al., 2003) and B (chiB) (PDB code: 1O6I) (Houston et al., 2002) from the bacterium Serratia marcescens. An additional $\alpha / \beta$ domain, composed of five antiparallel $\beta$-strands and two $\alpha$-helices, is inserted in the loop between strand $\beta 9$ and helix $\alpha 8$, and this domain might give the active site a groove character. Like all other Family 18 chitinases, CrChil has the DXDXE motif at the end of strand $\beta 5$, with Glu174 as the catalytic residue in the middle of the open end of the $(\beta / \alpha)_{8}$ barrel (Fig. 2a). Chitinase CrChil showed overall sequence similarities ranging from $24.2 \%$ to $54.8 \%$ to five other chitinases (afChi, cmChi, huChi, ChiA and $\mathrm{ChiB})$. However, the amino acid residues around the substrate-binding site and the catalytic centre were very conserved (Fig. 2a). When the structures afChi, cmChi, huChi, ChiA and ChiB were superimposed onto CrChil using the SSM method (Fig. 2b), we found that the core parts of all these structures had similar folding patterns, with the RMS deviation all between 0.6 and $1.3 \AA$ (Fig. 2b). Their main differences were in the $\mathrm{N}$ - and $\mathrm{C}$-terminal domains (Fig. 2a, b). Together, these results showed that 


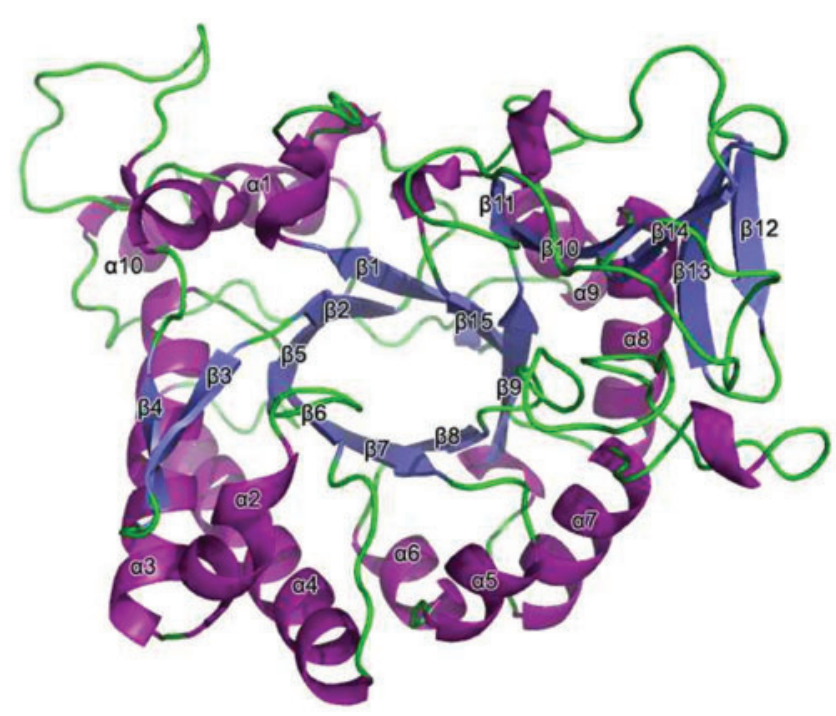

Fig. 1. Overall structure of CrChi1. Helices, strands and coil are coloured purple, slate and green, respectively. All secondary structural elements are labelled.

while the chitinases from different organisms shared low sequence similarity (Fig. 2a), their structures were very conserved (Fig. 2b), especially for the amino acid residues corresponding to the substrate-binding domain and the catalytic domain (Fig. 2a). Our analyses suggest that these chitinases probably share a common catalytic mechanism.

\section{Inhibitor binding site}

The sequence alignment (Fig. 2a) and structure comparison among the chitinases (Fig. 2b) suggested that CrChil might have the same active site as the other five homologues. Therefore, caffeine, a novel Family 18 chitinase inhibitor (Rao et al., 2005b; Schüttelkopf et al., 2006), was selected for a co-crystallization experiment to investigate the active site of the enzyme and the potential inhibitory mechanism of caffeine on CrChil.

The effectiveness of caffeine as an inhibitor of CrChil was initially approximated by measuring the activity of $2 \mathrm{nM}$ enzyme with $20 \mathrm{mM}$ substrate in the presence of different concentrations of caffeine. The results indicated that $1 \mathrm{mM}$ caffeine reduced the activity by about $50 \%$ (Fig. 3a). Complete steady-state kinetic parameters were then measured for the enzyme in the presence of caffeine using the modified fluorescent assay, and the results are shown in Fig. $3(\mathrm{~b})$. The $K_{\mathrm{m}}$ value of CrChil for the fluorescent oligosaccharide substrate 4 -methylumbelliferyl $\beta$-D- $N, N^{\prime}, N^{\prime \prime}$-triacetylchitotrioside was $9.5 \pm 0.3 \mathrm{mM}$. The nature of the inhibition is shown in Fig. 3(b); the double reciprocal plot suggests that caffeine behaves as a competitive inhibitor. The $K_{\mathrm{i}}$ of wild-type CrChil for caffeine was $19.7 \pm 0.3 \mathrm{mM}$.

A soaking experiment of CrChil with caffeine was carried out, followed by collection of diffraction data. The structure of the CrChi1-caffeine complex was solved and refined against $1.6 \AA$ resolution of the X-ray diffraction data (Table 1). The structure of CrChil in complex with caffeine defined the active centre of CrChil (Fig. 4a). In general, family 18 chitinases bind to their substrates in an extended recognition site. By convention, the sugars on the non-reducing end of the substrate are given negative numbers, and those on the reducing side are given positive numbers (Fig. 4a). In this convention, the scissile glycosidic bond lies between the -1 and +1 subsites. Previous structural studies of afChi, cmChi, huChi, ChiA and $\mathrm{ChiB}$ and their substrate-inhibitor complexes defined the GlcNAc subsites in the enzyme and they were found to form a deep groove on the surface of the protein. Similar properties were also observed in the CrChil structure (Fig. $4 a)$. The groove consists of six sugar-binding subsites, numbered from -4 (non-reducing end) to +2 (reducing end), with hydrolysis taking place on the glycosidic bond between the -1 and +1 subsites (van Aalten et al., 2001; Fusetti et al., 2002). The subsites -2 to +2 formed a deep groove, lined by side chains that are highly conserved in Family 18 chitinases, including the conserved DXDXE motif. In the -1 subsite, Asp172/Glu174 in CrChil are equivalent to Asp155/Glu157 in ScCTS1 (Saccharomyces cerevisiae) (Hurtado-Guerrero \& van Aalten, 2007), and to Asp125/Glu127 in hevamine (Terwisscha van Scheltinga et al., 1995), respectively. These are located at the end of $\beta 5$ and form the conserved DXDXE motif in Family 18 chitinases. As in other Family 18 chitinases, Glu174 is the catalytic residue, with Asp172 stabilizing the oxazolinium ring of the reaction intermediate. Trp381 is the most conserved residues in all Family 18 chitinases, forming the bottom of the -1 subsite, with some conserved residues forming the sidewall.

The caffeine inhibitor binds to CrChil in a position equivalent to the allosamidin allosamizoline moiety (Rao et al., 2005a). Allosamidin is a natural pseudotrisaccharide inhibitor of Family 18 chitinases and binds to subsites -3 to -1 in complex with chitinases (Rao et al., 2005a; van Aalten et al., 2001). This allosamizoline moiety is known to mimic the oxazolinium ion reaction intermediate formed upon nucleophilic attack of the $N$-acetyl oxygen on the anomeric carbon (van Aalten et al., 2001). The methylxanthine core makes similar interactions with the chitinases. In addition to the methylxanthine in the -1 subsite, an additional ordered inhibitor molecule was also observed.

The interactions between the caffeine moiety and the enzyme are similar to those observed in the afChiB1caffeine complex and the afChiB1-C2-dicaffeine complex (Rao et al., 2005b; Schüttelkopf et al., 2006). The active-site Asp172 points down into the catalytic core. The electrondensity map shows that the primary caffeine moiety is sandwiched between Trp134 and Trp381, and accepts two hydrogen bonds, one from the backbone amide of Trp134 and the other from the hydroxyl of Tyr242 (Fig. 4b). The 
(a)

afchiB1

huchi

Chis

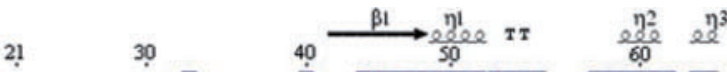

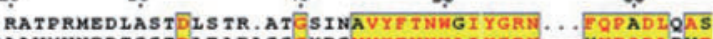

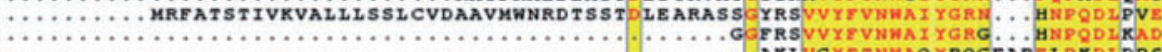

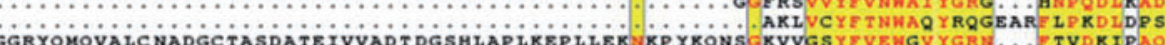
.................................... MSTRKAVIGYYFIPTNQINAYTETDT SVVPFPVSSITPAKAK

2 $\underset{70}{\stackrel{\beta 2}{r} \longrightarrow} \mathrm{rr}$

vin.

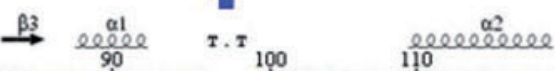

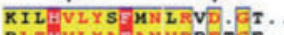

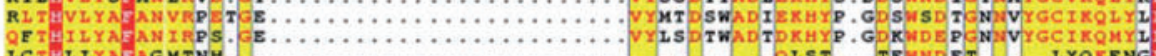

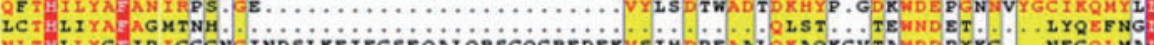

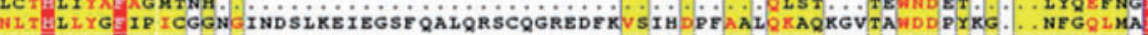

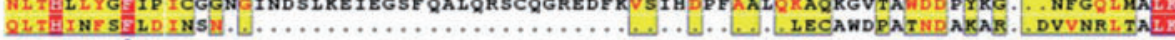

Cho

CrChi
afChiBi
cmchi
huChi
ChiA
ChiB

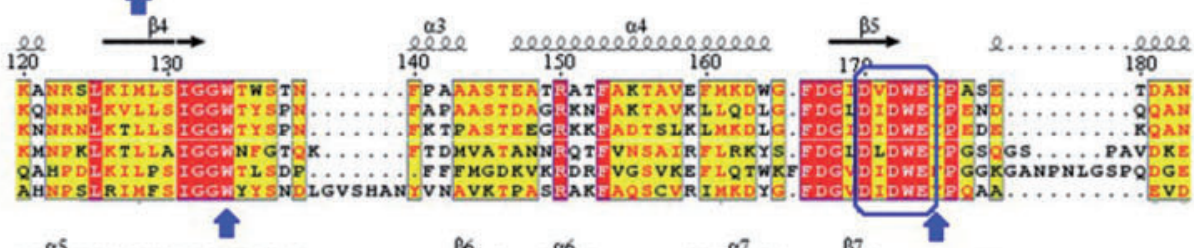

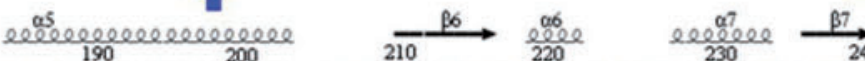

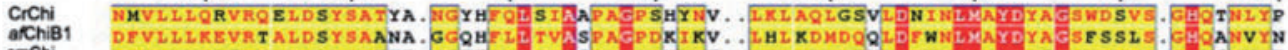

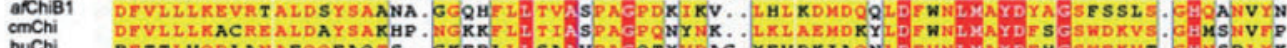

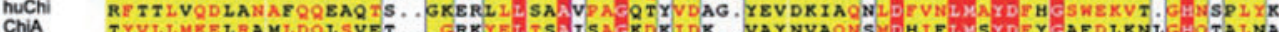

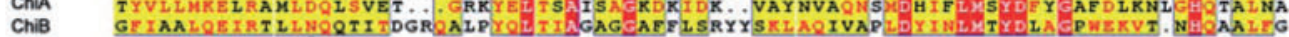
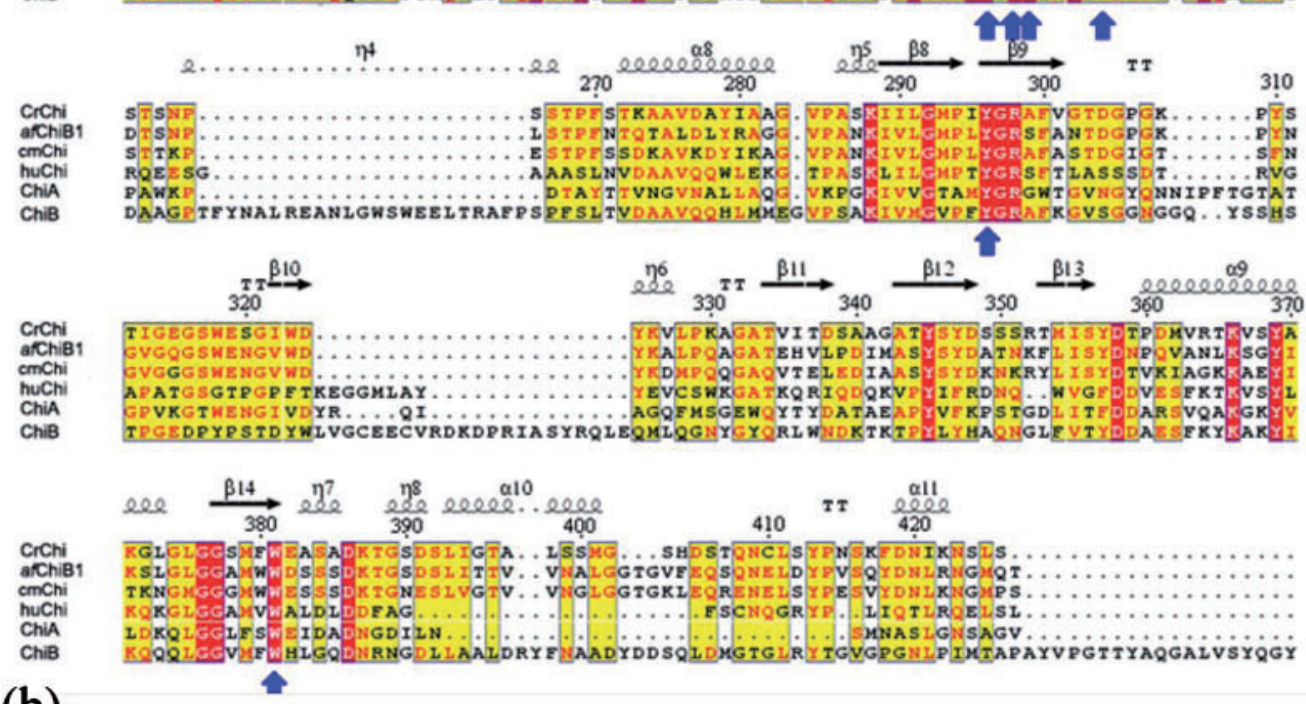

(b)

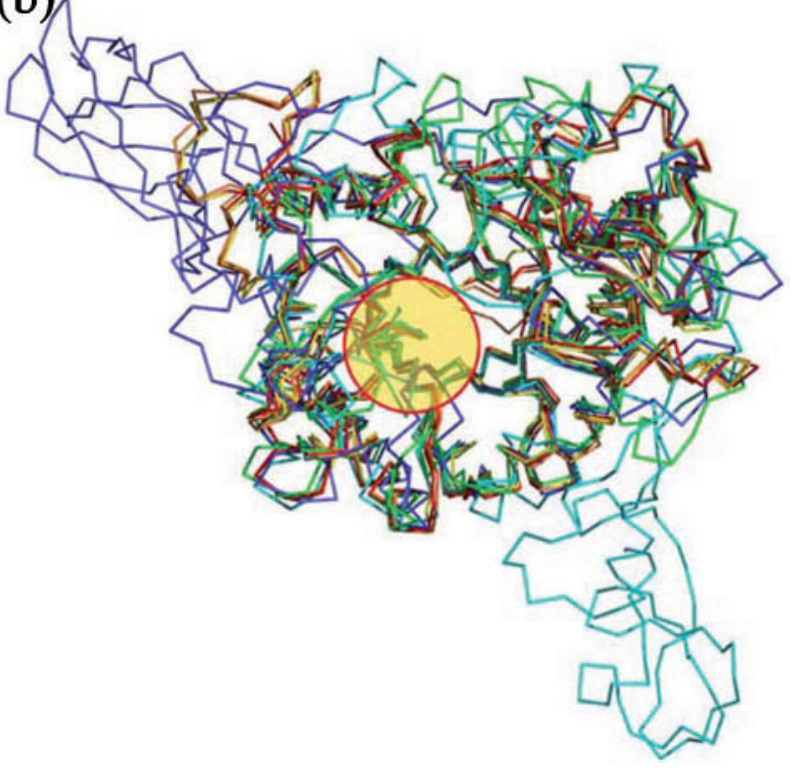

Glu174

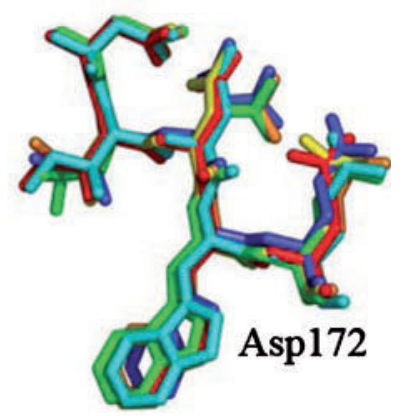

Trp173 
Fig. 2. (a) Structure-based sequence alignment between CrChi1 and homologues from different organisms. A red background indicates conserved residues; a yellow background indicates residues identified to be more than $80 \%$ conserved. The key DXDXE motif and residues important for inhibitor binding are marked by a blue frame and arrows, respectively. (b) Superposition of CrChi1 (gold), afChi1 (orange), huChi (green), ChiA (blue), ChiB (cyan) and cmChi (red). All the structures are shown as ribbons. The key residues in the active site (circled area, enlarged on the right) are shown as coloured sticks with the same colour scheme as the overall structure.

molecular interaction between CrChil and caffeine is described in Table 2.

\section{Mutagenesis and enzymology}

The structure shown in Fig. 4(b) suggests that residues Tyr46, Trp134 and Tyr242 in CrChil play important roles in the formation of hydrogen bonds between the enzyme and caffeine. To obtain an insight into how the conserved residues are involved in the binding, we conducted a mutagenesis study of CrChil. Table 3 shows the kinetic constants and $K_{\mathrm{i}}$ values for the wild-type CrChil as well as

(a)
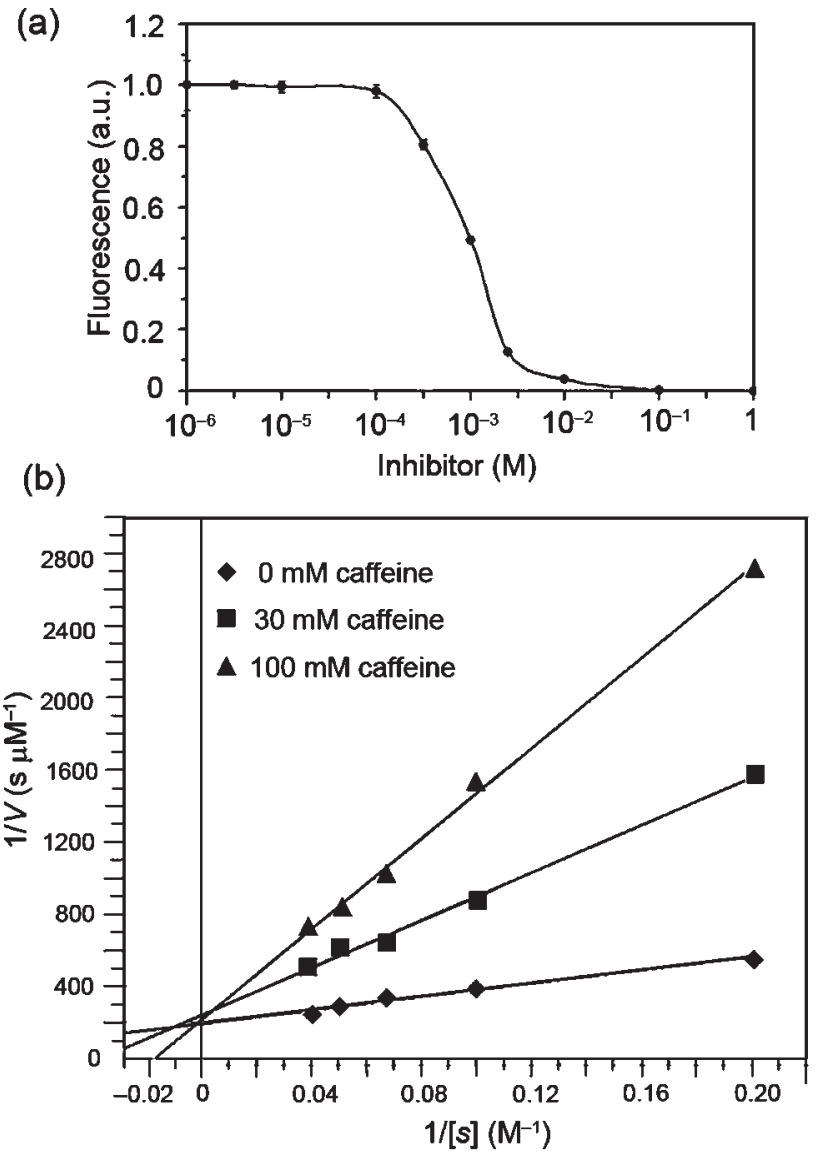

Fig. 3. (a) Dose-response curve for the caffeine inhibitor measured against CrChi1. (b) Lineweaver-Burk plots showing the effect of different concentrations of caffeine in the standard assay with 4-methylumbelliferyl $\beta$-D- $N, N^{\prime}, N^{\prime \prime}$-triacetylchitotrioside as substrate. A fit of all the data against a competitive inhibition model resulted in a $K_{\mathrm{m}}$ of $9.5 \pm 0.3 \mathrm{mM}$ and a $K_{\mathrm{i}}$ of $19.7 \pm 0.3 \mathrm{mM}$. its mutant derivatives. Caffeine showed competitive inhibitory activity in all cases.

The amino acid substitutions caused a decrease of over 1000 -fold in the enzymic activity. Two mutations, E174Q and $\mathrm{W} 134 \mathrm{G}$, completely inactivated the enzyme, confirm-

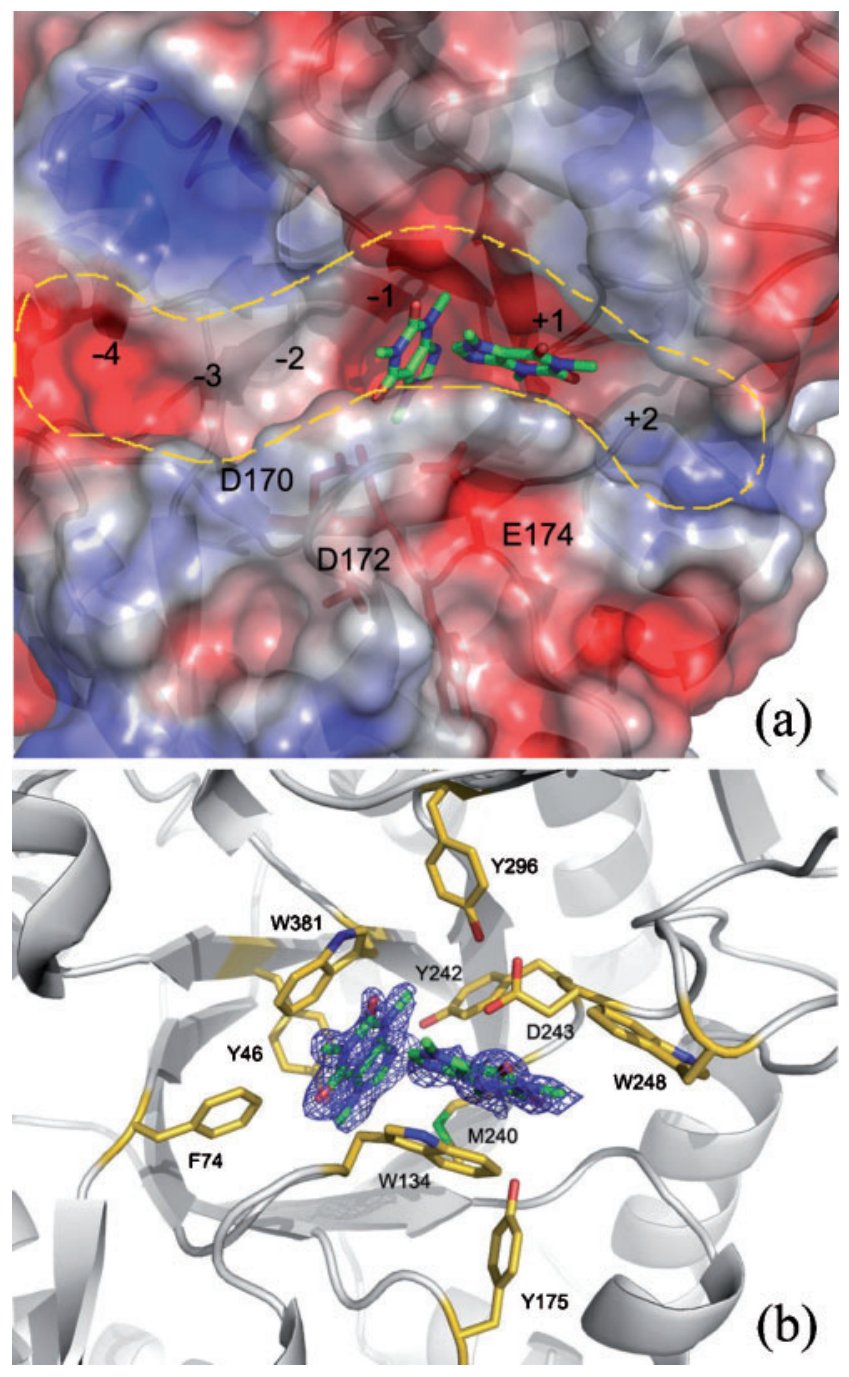

Fig. 4. (a) The crystal structure of caffeine in complex with $\mathrm{CrChi} 1$ is shown as a transparent surface. The substrate-binding cleft is outlined in yellow. The two caffeine molecules are shown in stick representation. Three important residues (Asp170, Asp172 and Glu174) from the Family 18 chitinase DXDXE motif are shown. (b) Caffeine bound to active site of CrChi1. The two bound caffeine molecules are shown as green sticks and covered with an omit density map at $1.1 \sigma$. The residues that are important in forming the binding pocket and in caffeine binding are shown as gold sticks. 
Table 2. Molecular interactions between $\mathrm{CrChi} 1$ and the inhibitor caffeine

\begin{tabular}{|c|c|c|}
\hline $\begin{array}{l}\text { Atom in } \\
\text { caffeine }\end{array}$ & Atom in CrChil & Distance $(\AA)$ \\
\hline \multicolumn{3}{|c|}{ Caffeine 1} \\
\hline $\mathrm{C} 5$ & $\mathrm{M} 240 \mathrm{~S}^{\delta}$ & 3.1 \\
\hline \multirow[t]{2}{*}{ C6 } & $\mathrm{D} 172-\mathrm{O}^{\delta 2}$ & 3.0 \\
\hline & $\mathrm{E} 174-\mathrm{O}^{\varepsilon 1}$ & 2.9 \\
\hline N3 & Y242-OH & 2.7 \\
\hline $\mathrm{O} 1$ & Wat453-D243-O ${ }^{\delta 2}$ & $2.8-2.7$ \\
\hline $\mathrm{O} 2$ & W134-N & 2.9 \\
\hline \multicolumn{3}{|c|}{ Caffeine 2} \\
\hline $\mathrm{N} 2$ & $\mathrm{D} 243-\mathrm{O}^{\delta 2}$ & 3.0 \\
\hline $\mathrm{C} 2$ & $\mathrm{D} 243-\mathrm{O}^{\delta 2}$ & 2.9 \\
\hline $\mathrm{O} 1$ & Wat258-Y242-O & 2.7 \\
\hline
\end{tabular}

ing the essential roles of these two residues in the catalytic process. The Glu174 residue was considered to act as a proton donor in the catalysis. Trp134 is located in the substrate-binding domain and forms a hydrogen bond with caffeine (Fig. 4b, Table 2). The benzene ring of Trp134 may also be important for the formation and maintenance of the substrate-binding sites. Based on sequence comparisons (Fig. 2a), Glu174 and Trp134 are conserved in all Family 18 chitinases examined. The loss of function of E174Q mutation in CrChil is also consistent with the importance of the acid residue at that position, similar to a previous finding for the E144Q mutation of ChiB from $S$. marcescens (Houston et al., 2002). The W134G mutant showed no detectable fluorescence signal and showed no detectable binding in the tryptophan fluorescence binding experiments, similar to results reported previously (Rao et al., 2005a). Tyr242 is also conserved in Family 18 chitinases, and our site-directed mutagenesis of Tyr242 in CrChil impaired the enzymic activity, although the activity was not completely eliminated. Residue Tyr242 forms hydrogen bonds with caffeine. Changing residue Tyr242 to Gly or Phe reduced $k_{\text {cat }}$ by three orders of magnitude, while no large effects on $K_{\mathrm{m}}$ were observed (Table 3 ). This result suggests that this residue is crucial for catalysis rather than substrate binding, in agreement with mutational studies of this residue in other Family 18 chitinases (Bortone et al.,
2002; Rao et al., 2005a; van Aalten et al., 2001). Tyr46 is also highly conserved in Family 18 chitinases; the enzymic activity was impaired when Tyr46 in CrChil was mutated (Table 3). Tyr46 is located near the substrate-binding domain (Fig. 4b), which suggests that it may influence substrate binding. Interestingly, when Tyr46 was changed to phenylalanine, the protein was expressed as inclusion bodies in E. coli, suggesting that this residue may be required for proper structure formation. Moreover, this mutation caused a reduction of $k_{\text {cat }}$ by three orders of magnitude after renaturation and refolding of CrChil, but had a relatively small effect on $K_{\mathrm{m}}$ (Table 3 ). These results suggest that mutation of Tyr46 affected the catalytic activity and resulted in a decrease of $k_{\text {cat, }}$ while the substrate binding was not affected.

\section{Conclusions}

There is growing concern about various problems caused by widespread use of chemical pesticides, including environmental pollution and long-term persistence of residues. As a result, increasing efforts have been paid to developing biological control agents. Nematophagous fungi have been proposed as biological agents to control harmful nematodes because of their unique ability to infect and kill the nematodes (Åhman et al., 2002; Gan et al., 2007a; Yang et al., 2007). In this study, we determined the crystal structure of chitinase CrChil from the nematophagous fungus $C$. rosea. We identified active-site residues based on the crystal structure of the apoenzyme complexed with caffeine. Our results should help understand the catalytic mechanism of fungal chitinases involved in the infection of hosts, and the information presented here could be further exploited for designing more effective pesticides, fungicides and anti-malarial drugs.

\section{ACKNOWLEDGEMENTS}

We are grateful to Professor Jianping Xu (McMaster University, Canada) for his valuable comments and critical discussions. This work was funded by National Basic Research Program of China (approved no. 2007CB411600), by projects from the National Natural Science Foundation of China (approved nos. 30630003, 30960229, 30860278 and 30870486), the Department of Science and Technology of Yunnan Province (approved nos. 2007C007Z, 2006C0071M,

Table 3. Mutagenesis of Crchi1 and enzymological data for the wild-type and mutant proteins

All experiments were performed in triplicate. ND, No significant signal.

\begin{tabular}{|llcccc|}
\hline Mutation & Protein & $\boldsymbol{K}_{\mathrm{m}}(\boldsymbol{\mu M})$ & $\boldsymbol{V}_{\max }\left(\boldsymbol{\mu} \mathbf{M ~ s}^{-\mathbf{1}}\right)$ & $\boldsymbol{k}_{\mathrm{cat}}\left(\mathbf{s}^{-\mathbf{1}}\right)$ & $\boldsymbol{k}_{\mathrm{cat}} / \boldsymbol{K}_{\mathrm{m}}\left(\boldsymbol{\mu} \mathbf{M}^{-\mathbf{1}} \mathbf{s}^{-\mathbf{1}}\right)$ \\
\hline Control & Soluble & $9.5 \pm 0.3$ & $0.005 \pm 0.00007$ & $2.59 \pm 0.03$ & 0.27 \\
Y46F & Insoluble & $11.5 \pm 1.0$ & $0.001 \pm 0.00003$ & $0.0062 \pm 0.0002$ & 0.0005 \\
W134G & Soluble & $\mathrm{ND}$ & $\mathrm{ND}$ & $\mathrm{ND}$ & $\mathrm{ND}$ \\
E174Q & Soluble & $\mathrm{ND}$ & $\mathrm{ND}$ & $0.0054 \pm 0.0002$ & ND \\
Y242F & Soluble & $10.8 \pm 2.4$ & $0.001 \pm 0.00003$ & $0.005 \pm 0.0005$ & 0.0005 \\
Y242G & Soluble & $6.9 \pm 1.0$ & $0.0009 \pm 0.0001$ & 0.0008 \\
\hline
\end{tabular}


2007C0001R and 2009CI052), and the Tsinghua University Initiative Scientific Research Program (approved no. 2009THZ01).

\section{REFERENCES}

Åhman, J., Johanson, T., Olsson, M., Punt, P. J., van den Hondel, C. A. M. J. J. \& Tunlid, A. S. (2002). Improving the pathogenicity of a nematode-trapping fungus by genetic engineering of a subtilisin with nematotoxic activity. Appl Environ Microbiol 68, 3408-3415.

Arai, N., Shiomi, K., Yamaguchi, Y., Masuma, R., Iwai, Y., Turberg, A., Koelbl, H. \& Omura, S. (2000). Argadin, a new chitinase inhibitor, produced by Clonostachys sp. FO-7314. Chem Pharm Bull (Tokyo) 48, 1442-1446.

Bortone, K., Monzingo, A. F., Ernst, S. \& Robertus, J. D. (2002). The structure of an allosamidin complex with the Coccidiodes immitis defines a role for second acid residue in substrate assisted mechanism. J Mol Biol 320, 293-302.

Brünger, A. T., Adams, P. D., Clore, G. M., DeLano, W. L., Gros, P., Grosse-Kunstleve, R. W., Jiang, J. S., Kuszewski, J., Nilges, M. \& other authors (1998). Crystallography \& NMR System: a new software suite for macromolecular structure determination. Acta Crystallogr D Biol Crystallogr 54, 905-921.

DeLano, W. (2002). The PyMol Molecular Graphics System. San Carlos, CA, USA: DeLano Scientific.

Emsley, P. \& Cowtan, K. (2004). Coot: model-building tools for molecular graphics. Acta Crystallogr D Biol Crystallogr 60, 2126-2132.

Esnouf, R. M. (1997). An extensively modified version of MolScript that includes greatly enhanced coloring capabilities. J Mol Graph Model 15, 132-134.

Fusetti, F., von Moeller, H., Houston, D., Rozeboom, H. J., Dijkstra, B. W., Boot, R. G., Aerts, J. M. \& van Aalten, D. M. F. (2002). Structure of human chitotriosidase. Implications for specific inhibitor design and function of mammalian chitinase-like lectins. J Biol Chem 277, 25537-25544.

Gan, Z. W., Yang, J. K., Tao, N., Liang, L. M., Mi, Q. L., Li, J. \& Zhang, K. Q. (2007a). Cloning of the gene Lecanicillium psalliotae chitinase Lpchil and identification of its potential role in the biocontrol of root-knot nematode Meloidogyne incognita. Appl Microbiol Biotechnol 76, 1309-1317.

Gan, Z. W., Yang, J. K., Tao, N., Yu, Z. F. \& Zhang, K. Q. (2007b). Cloning and expression analysis of a chitinase gene Crchil from the mycoparasitic fungus Clonostachys rosea (syn. Gliocladium roseum). J Microbiol 45, 422-430.

Gan, Z. W., Yang, J. K., Tao, N., Lou, Z. Y., Mi, Q. L., Meng, Z. H. \& Zhang, K. Q. (2009). Crystallization and preliminary crystallographic analysis of a chitinase from Clonostachys rosea. Acta Crystallogr Sect F Struct Biol Cryst Commun 65, 386-388.

Gooday, G. W., Zhu, W. Y. \& O'Donnell, R. W. (1992). What are the roles of chitinases in the growing fungus. FEMS Microbiol Lett $\mathbf{1 0 0}$ 387-391.

Hollis, T., Monzingo, A. F., Bortone, K., Ernst, S., Cox, R. \& Robertus, J. D. (2000). The X-ray structure of a chitinase from the pathogenic fungus Coccidioides immitis. Protein Sci 9, 544-551.

Houston, D. R., Eggleston, I., Synstad, B., Eijsink, V. G. \& van Aalten, D. M. F. (2002). The cyclic dipeptide C1-4[cyclo-(L-Arg-D-Pro)] inhibits family 18 chitinases by structural mimicry of a reaction intermediate. Biochem J 368, 23-27.

Hurtado-Guerrero, R. \& van Aalten, D. M. F. (2007). Structure of Saccharomyces cerevisiae chitinase 1 and screening-based discovery of potent inhibitors. Chem Biol 14, 589-599.
Kuranda, M. J. \& Robbins, P. W. (1991). Chitinase is required for cell separation during growth of Saccharomyces cerevisiae. J Biol Chem 266, 19758-19767.

Laskowski, R., MacArthur, M., Moss, D. \& Thornton, J. (1993). PROCHECK: a program to check the stereochemical quality of protein structures. J Appl Crystallogr 26, 283-291.

Li, J., Yang, J. K., Huang, X. W. \& Zhang, K. Q. (2006). Purification and characterization of an extracellular protease from Clonostachys rosea and its potential as a pathogenic factor. Process Biochem 41, 925929.

Lorito, M., Mach, R. L., Sposato, P., Strauss, J., Peterbauer, C. K. \& Kubicek, C. P. (1996). Mycoparasitic interaction relieves binding of the Crel carbon catabolite repressor protein to promoter sequences of the ech42 (endochitinase-encoding) gene in Trichoderma harzianum. Proc Natl Acad Sci U S A 93, 14868-14872.

Mamarabadi, M., Jensen, B. \& Lübeck, M. (2008). Three endochitinase-encoding genes identified in the biocontrol fungus Clonostachys rosea are differentially expressed. Curr Genet 54, 57-70.

Matthews, B. W. (1968). Solvent content of protein crystals. J Mol Biol 33, 491-497.

McCoy, A. J., Grosse-Kunstleve, R. W., Adams, P. D., Winn, M. D., Storoni, L. C. \& Read, R. J. (2007). Phaser crystallographic software. J Appl Crystallogr 40, 658-674.

Meng, Z. H., Lou, Z. Y., Liu, Z., Hui, D., Bartlam, M. \& Rao, Z. H. (2006). Purification, characterization, and crystallization of human pyrroline5-carboxylate reductase. Protein Expr Purif 49, 83-87.

Merritt, E. A. \& Murphy, M. E. (1994). Raster3D version 2.0. A program for photorealistic molecular graphics. Acta Crystallogr D Biol Crystallogr 50, 869-873.

Omura, S., Arai, N., Yamaguchi, Y., Masuma, R., Iwai, Y., Namikoshi, M., Turberg, A., Kölbl, H. \& Shiomi, K. (2000). Argifin, a new chitinase inhibitor, produced by Gliocladium sp. FTD-0668. J Antibiot (Tokyo) 53, 603-608.

Otwinowski, Z. \& Minor, W. (1997). Processing of X-ray diffraction data collected in oscillation mode. Methods Enzymol 276, 307326.

Papanikolau, Y., Tavlas, G., Vorgias, C. E. \& Petratos, K. (2003). De novo purification scheme and crystallization conditions yield highresolution structures of chitinase A and its complex with the inhibitor allosamidin. Acta Crystallogr D Biol Crystallogr 59, 400-403.

Rajamohan, F., Alzate, O., Cotrill, J. A., Curtiss, A. \& Dean, D. H. (1996). Protein engineering of Bacillus thuringiensis $\delta$-endotoxin: mutations at domain II of CrylAb enhance receptor affinity and toxicity towards gypsy moth larvae. Proc Natl Acad Sci U S A 93, 14338-14343.

Rao, F. V., Andersen, O. A., Vora, K. A., DeMartino, J. A. \& van Aalten, D. M. F. (2005a). Methylxanthine drugs are chitinase inhibitors: investigation of inhibition and binding modes. Chem Biol 12, 973980.

Rao, F. V., Houston, D. R., Boot, R. G., Aerts, J. M., Hodkinson, M., Adams, D. J., Shiomi, K., Omura, S. \& van Aalten, D. M. F. (2005b). Specificity and affinity of natural product cyclopentapeptide inhibitors against A. fumigatus, human, and bacterial chitinases. Chem Biol $12,65-76$.

Schüttelkopf, A. W., Andersen, O. A., Rao, F. V., Allwood, M., Lloyd, C., Eggleston, I. M. \& van Aalten, D. M. F. (2006). Screening-based discovery and structural dissection of a novel family 18 chitinase inhibitor. J Biol Chem 281, 27278-27285.

Seidl, V., Huemer, B., Seiboth, B. \& Kubicek, C. P. (2005). A complete survey of Trichoderma chitinases reveals three distinct subgroups of family 18 chitinases. FEBS J 272, 5923-5939. 
Sutton, J. C., Li, D. W., Peng, G., Yu, H., Zhang, P. \& ValdebenitoSanhueza, R. M. (1997). Gliocladium roseum a versatile adversary of a Botrytis cinerea in crops. Plant Dis 81, 316-328.

Terwisscha van Scheltinga, A. C., Armand, S., Kalk, K. H., Isogai, A., Henrissat, B. \& Dijkstra, B. W. (1995). Stereochemistry of chitin hydrolysis by a plant chitinase/lysozyme and X-ray structure of a complex with allosamidin: evidence for substract assisted catalysis. Biochemistry 34, 15619-15623.

van Aalten, D. M. F., Komander, D., Synstad, B., Gàseidnes, S., Peter, M. G. \& Eijsink, V. G. H. (2001). Structural insights into the catalytic mechanism of a family 18 exo-chitinase. Proc Natl Acad Sci U S A 98, 8979-8984.

Xue, A. G. (2003). Biological control of pathogens causing root rot complex in field pea using Clonostachys rosea strain ACM941. Phytopathology 93, 329-335.

Yang, J. K., Tian, B. Y., Liang, L. M. \& Zhang, K. Q. (2007). Extracellular enzymes and the pathogenesis of nematophagous fungi. Appl Microbiol Biotechnol 75, 21-31.

Edited by: M. Tien 